Cahiers québécois de démographie

\title{
La décroissance de la population du Québec
}

Si on peut la prévoir, peut-on la prévenir?

\section{Quebec's Population Decline}

If It is foreseeable, is It Avoidable?

\section{El decrecimiento de la población del Québec Podemos preverla, podemos prevenirla?}

\section{Jeanine Perreault, Rosalinda Costa et M. V. George}

Volume 15, numéro 2, octobre 1986

La décroissance démographique et ses implications

URI : https://id.erudit.org/iderudit/600595ar

DOI : https://doi.org/10.7202/600595ar

Aller au sommaire du numéro

Éditeur(s)

Association des démographes du Québec

ISSN

0380-1721 (imprimé)

1705-1495 (numérique)

Découvrir la revue

Citer cet article

Perreault, J., Costa, R. \& George, M. V. (1986). La décroissance de la population du Québec : si on peut la prévoir, peut-on la prévenir? Cahiers québécois de démographie, 15(2), 213-225. https://doi.org/10.7202/600595ar
Résumé de l'article

La fécondité du Québec est la plus faible parmi toutes les provinces canadiennes, et selon toute vraisemblance la population du Québec s'achemine vers la décroissance. Dans cet article, les auteurs tentent d'expliciter certains des scénarios possibles qui pourraient empêcher cette décroissance. Dans un premier temps, ils présentent brièvement les tendances actuelles et précisent les hypothèses retenues pour la simulation. Puis, ils examinent les résultats en situant la décroissance dans le temps et en définissant l'ordre de grandeur de la population maximale. Et finalement, ils présentent certains des scénarios de rechange qui pourraient empêcher la décroissance. 
Cahiers québécois de démographie

Vol. 15, no 2, octobre 1986

\title{
La décroissance de la population du Québec: si on peut la prévoir, peut-on la prévenir?
}

\author{
Jeanine PERREAULT, Rosalinda COSTA et M.V. GEORGE*
}

\section{INTRODUCTION}

La décroissance démographique des sociétés occidentales, à plus ou moins brève échéance, apparaît de plus en plus probable. Selon toute vraisemblance, la population du Québec s'achemine vers un déclin : au tournant du siècle, si les tendances actuelles se poursuivent, quelque dix ans plus tard, vers 2010, si la fécondité et la migration se stabilisent à leur niveau actuel. Seule une remontée de la fécondité ou une immigration très forte pourrait empêcher ce déclin. En cela, le Québec ne s'écarte pas de la tendance observée presque partout dans les pays industrialisés. Si le Québec a une légère avance sur les provinces voisines, par contre d'autres pays le devancent. Nous pensons à 1'Allemagne de l'Ouest par exemple, qui a déjà atteint un indice synthétique de fécondité de 1,3 enfant par femme.

Si 1 'on peut prévoir la décroissance, peut-on la prévenir ? A cette question cruciale, lourde de conséquences, posée très souvent aux démographes, nous allons tenter ici d'apporter des éléments de réponse. Nous n'aborderons cependant pas des questions d'ordre théorique, comme celle de savoir si $1^{\prime}$ on doit prévenir le déclin, ou encore, celle de déterminer le niveau "optimal" de la population du Québec.

La démarche adoptée consiste essentiellement à expliciter certains des scénarios possibles qui pourraient empêcher $1 a$ décroissance de la population québécoise (c'est-à-dire, qui assureraient un taux de croissance nul, plutôt que négatif), en agissant tantôt sur la fécondité, tantôt sur la migration, les deux facteurs de croissance démographique. L'horizon choisi est l'année 2026 (période 1986-2026), soit un quart de siècle après 1 'apparition du déclin dans 1 'hypothèse du prolongement des tendances. Pour chacun des scénarios considérés, nous situons

Statistique Canada, Division de la démographie, Ottawa.

Les vues exprimées dans ce document sont celles des auteurs et ne représentent pas nécessairement celles de Statistique Canada. 
d'abord le déclin dans le temps (quand apparaît-il selon diverses hypothèses), puis nous définissons l'ordre de grandeur de la population québécoise à ce moment-là, et finalement nous présentons certains des scénarios de "rechange» qui pourraient empêcher le déclin. Voyons d'abord très brièvement les tendances actuelles avant de préciser les scénarios retenus.

\section{LES TENDANCES ACTUELLES \\ La fécondité}

La fécondité du Québec, chacun le sait, est la plus faible parmi toutes les provinces canadiennes. En 1985, I'indice synthétique de fécondité atteignait 1,42 enfant par femme (chiffres préliminaires fournis par le Bureau de la statistique du Québec), une baisse de $2 \%$ par rapport à l'année précédente. L'indice était plus élevé pour l'ensemble du pays comme dans la province voisine de 1'Ontario : 1,69 (données pour 1984, Section de l'état civil, Statistique Canada). Le rythme de baisse de la fécondité a ralenti au Québec ces dernières années, mais la baisse continue : récemment, le rythme de baisse atteignait 3,3\% entre 1982 et $1983,1,4 \%$ entre 1983 et 1984 , et $2,1 \%$ entre 1984 et 1985. En Ontario par contre, la baisse est moins rapide, avec des pauses occasionnelles et même pour la dernière année, une faible remontée.

En termes de nombre annuel de naissances, l'indice synthétique de fécondité de 1,42 au Québec combiné à une structure par âge favorable a donné, en 1985, 86000 naissances (comparé à 88000 1 'année précédente).

\section{La migration}

Depuis 15 ans, la migration nette au Québec a généralement été négative, à cause du poids déterminant de la migration interprovinciale (négative), cette dernière faisant plus qu'annuler les gains de la migration internationale. En 1985-1986 (année censitaire), et ce pour la première fois en dix ans, le solde migratoire total (interprovincial et international) du Québec redevenait positif, renversant ainsi un mouvement négatif qui remonte au début des années soixante (chiffres établis à partir des fichiers des allocations familiales, données non encore publiées). Il est encore trop tôt pour se prononcer sur la signification de cette nouvelle situation, et $1^{\prime}$ on ne peut exclure à priori qu'il s'agisse là d'un changement d'ordre conjoncturel (entre autres, des retours de 1'Alberta). Néamoins, depuis 10 ans la perte annuelle de population due à la migration interprovinciale a constamment diminué, passant de -46000 en 
1977-1978 à -3000 en 1985-1986. Une situation d'équilibre dans les échanges migratoires avec les autres provinces est un scénario qui doit désormais être considéré plausible au même titre qu'un scénario de solde négatif. Quant au solde estimé des migrations internationales, $i l$ est toujours positif, quelque 9000 en 1985-1986, soit une immigration de 16000 et une émigration de 7 000. Il s'agit toutefois d'un niveau très faible, en baisse depuis plusieurs années, reflétant la politique d'immigration canadienne et la conjoncture économique récente. En termes relatifs, la proportion de l'ensemble des immigrants internationaux qui déclarent choisir le Québec comme province de destination change peu : quelque $18 \%$ des immigrants en 1985-1986. Le nombre d'émigrants internationaux pour sa part varie relativement peu : entre 7 et 10 mille par an depuis dix ans (mais il s'agit d'estimations plus fragiles que les autres).

\section{CONSTRUCTION DES SCÉNARIOS}

A la lumière de l'évolution démographique observée depuis les quinze dernières années, soit la décennie soixante-dix et la première moitié de la décennie quatre-vingt, et en tenant compte de la conjoncture actuelle, nous avons défini un jeu d'hypothèses de fécondité et de migration considérées "plausibles" pour les fins de notre exercice de simulation.

\section{Fécondité}

Cinq hypothèses de fécondité ont été retenues, soit une fourchette autour de la valeur courante, comportant deux hypothèses de poursuite de la baisse et deux hypothèses de remontée de la fécondité, plus l'hypothèse de constance au niveau récent. Le niveau constant a été fixé à $1,45,1 \mathrm{a}$ dernière estimation disponible lorsque ces simulations ont été faites (celle de 1'année 1984). Depuis, comme nous $1^{\prime}$ avons mentionné, l'indice synthétique de fécondité a encore diminué. L'écart maximum (à partir de 1996), entre deux hypothèses contiguës est de 0,2 enfant par femme; l'écart total (entre hypothèses extrêmes) est donc de 0,8 enfant par femme. Ces hypothèses sont présentées au graphique 1. Nous aurions pu, c'est évident, choisir des niveaux au delà de 1,9 et en deçà de 1,1; aucun n'est impossible en soi. Nous avons préféré le domaine du "plausible" à celui du "possible" (notion éminemment subjective, i.1 faut en convenir) à l'intérieur du cadre temporel défini dans l'introduction (horizon 2026 , soit 40 ans). 
Graphique 1

Indice synthétique de fécondité, Québec, 1966-2026

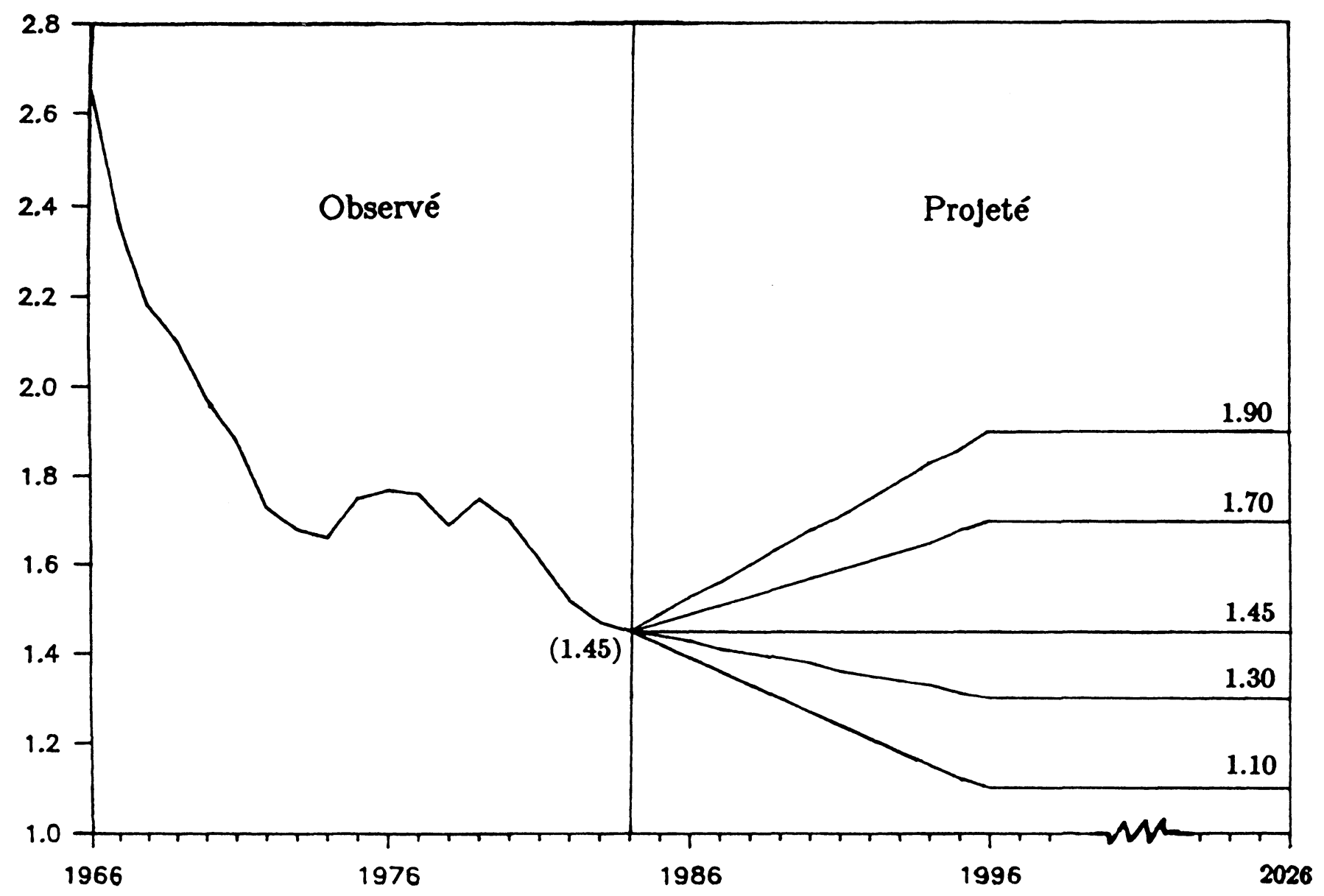

Source : La statistique de 1'état civil, volume 1, naissances et décès, catalogue 84-204 annuel, Statistiaue Canada. 1966-1984: pour les hypothèses d'avenir. consulter le texte. 


\section{Migration}

Cinq scénarios de migration ont été retenus : un scénario d'équilibre entre les entrées et sorties (migration nette zéro) lequel semble refléter à peu près la situation actuelle, deux scénarios négatifs de -10000 et -20000 , et deux scénarios de migration nette positive de +10000 et +20000 . Comme pour la fécondité, ce choix est subjectif. Si l'on exclut les deux années de 1977 à 1979 à cause de leur caractère particulier, la migration totale nette au Québec a fluctué entre -17000 et +5000 . Nous avons construit une fourchette autour du scénario d'équilibre en ajoutant une option positive de +20000 , option devenue "plausible" pour l'avenir, à la lumière des tendances très récentes. Ce scénario veut refléter à la fois une hausse de l'immigration internationale et une amélioration du solde migratoire interne.

Ces scénarios sont présentés au graphique 2. I'écart annuel maximum (dès 1986-1987) entre deux scénarios contigus est de 10000 migrants; l'écart maximum entre les deux scénarios extrêmes est donc de 40000 .

\section{PRÉSENTATION DES RÉSULTATS}

Les résultats de la simulation se présentent sous forme d'un ensemble de tableaux établis à partir de l'algorithme de projection de Statistique Canadal. Ces tableaux donnent la population par âge et sexe, pour chaque scénario retenu, ainsi que les composantes de la croissance (naissances, décès, migration) et autres indices de croissance.

Les résultats ont été regroupés en deux sous-ensembles, selon que c'est la fécondité ou la migration qui est l'objet de la simulation :

A.- Pour divers niveaux de fécondité donnés: l'ensemble des populations obtenues et des niveaux (annuels et cumulés) de migration nette nécessaires pour empêcher la décroissance.

1. L'algorithme a été modifié légèrement afin "d'empêcher" l'accroissement démographique de devenir négatif. Dans tous les scénarios retenus, le taux d'accroissement naturel diminue continuellement. Lorsque $I^{\prime}$ accroissement apparait négatif, une valeur égale à la "décroissancel est définie comme étant "le nombre de migrants ou de naissances nécessaires pour empêcher que la population ne diminuell. Ces migrants ou naissances viennent alors s'ajouter à la population. 
Graphique 2

Migration annuelle nette (en milliers), Québec, 1971-2.027

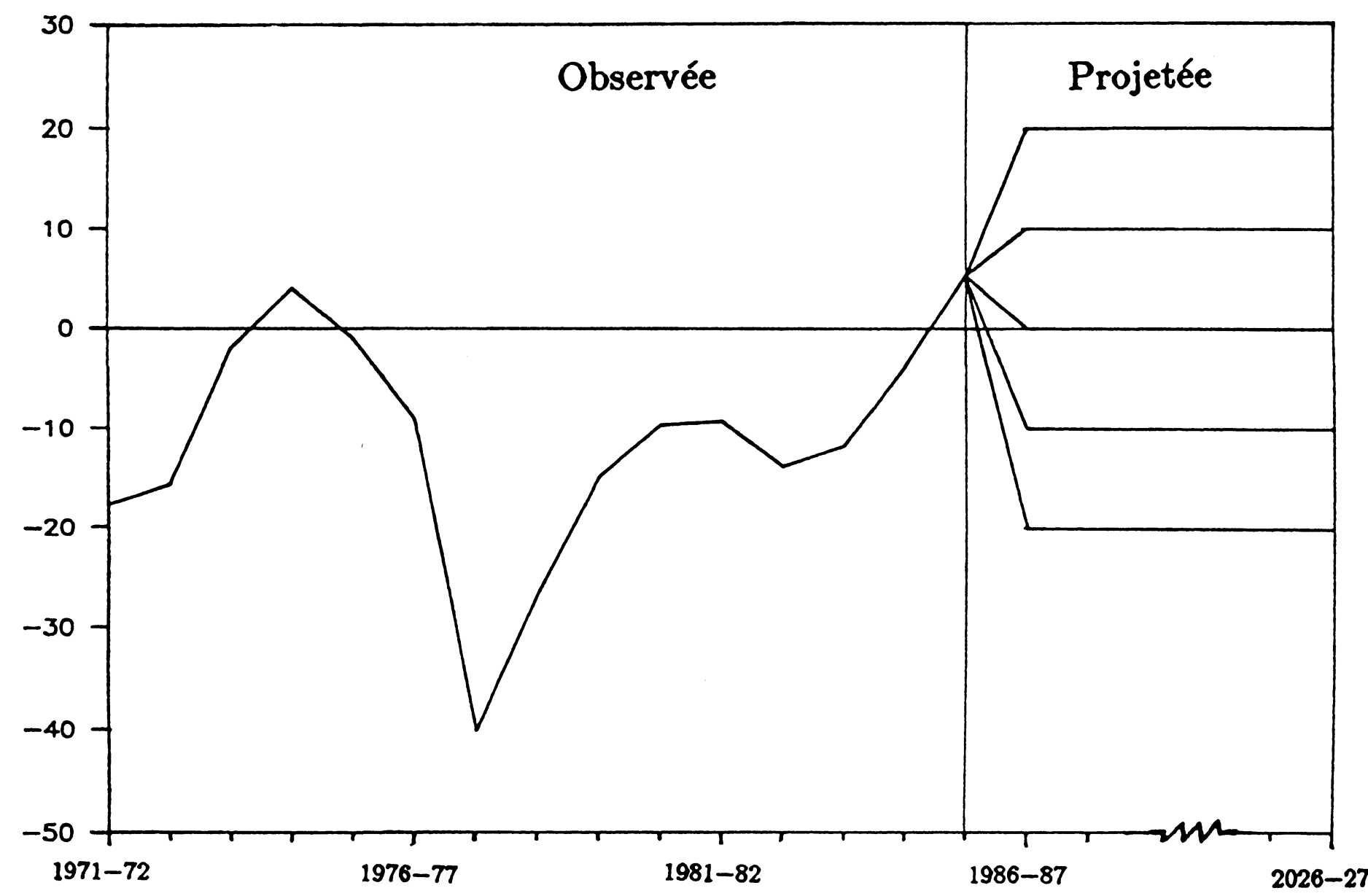
territoires, au ler juin 1985, volume 3, catalogue 91-210, Statistique Canada, 1971-1972 à 1984-1985; pour les hypothèses d'avenir, voir le texte. 
B.- Pour divers niveaux de migration nette donnés : 1 'ensemble des populations obtenues et les nombres de naissances annuelles nécessaires pour empêcher la décroissance.

Seuls les résultats du sous-ensemble A seront présentés ici, à savoir ceux portant sur "le nombre de migrants nets nécessaires pour empêcher que la population ne diminue", selon divers scénarios de fécondité.

3.1 Le déclin dans le temps et le nombre de migrants nets nécessaires pour contrer la décroissance

Le tableau et les graphiques qui suivent résument les résultats de la simulation. On y retrouve : a) 1'année où 1 'accroissement devient nul pour divers niveaux de fécondité, ceci marquant le début du déclin; b) le chiffre de population atteint à ce moment; et c) les nombres (annuels et cumulés) de migrants nets nécessaires pour contrer la décroissance.

Selon le tableau 1 , dans l'hypothèse de fécondité la plus faible, soit un indice synthétique de fécondité égal à 1,1 , la décroissance s'amorce avant la fin du siècle, dans dix ans. La population maximale atteinte se situe alors à 6,8 millions et la migration doit augmenter d'année en année pour contrer la décroissance. A 1 'horizon 2026, le nombre total de migrants nets cumulés sur 1 'ensemble de la période aurait atteint près d'un million. A droite du tableau, dans 1'hypothèse opposée d'une remontée de la fécondité à 1,9 , niveau inférieur mais qui se rapproche du seuil de remplacement des générations, la population québécoise continue d'augmenter jusque vers 2019-2020, pour atteindre environ 7,4 millions. Le nombre de migrants nets nécessaires est beaucoup plus faible, de l'ordre de 50 mille. Dans I'hypothèse intermédiaire, correspondant grosso modo à une extrapolation de la situation actuelle (avec un indice synthétique de fécondité égal à 1,45$)$, la décroissance apparaît vers 2006, dans vingt ans, et le nombre cumulé de migrants nets approche le demi-million à 1'horizon 2026. Le graphique 3 illustre de façon schématique ces divers résultats.

Les résultats de simulations correspondantes effectuées pour 1'Ontario et le Canada dans son ensemble montrent que le déclin n'apparaft pas avant 2026 en Ontario, dans $I^{\prime}$ 'hypothèse où $1 a$ fécondité et la migration actuelles sont maintenues constantes, la population atteignant alors près de 13 millions. Pour l'ensemble du Canada, dans la même hypothèse de constance des conditions actuelles (fécondité à 1,7 et migration nette de 65000 ), le déclin s'amorce vers 2026 et la population maximale atteinte serait de 1 'ordre de 31 millions. 
Tableau 1

Niveau de migration nette, annuel et cumulé, nécessaire pour empêcher la décrolssance, selon diverses hypothèses de fécondité. Québec, 1986-2026 (nombre en milliers)

\begin{tabular}{|c|c|c|c|c|c|c|c|c|c|c|c|c|c|c|c|}
\hline \multirow[b]{2}{*}{ Année } & \multicolumn{3}{|c|}{$I S F=1,1$} & \multicolumn{3}{|c|}{$I S F=1,3$} & \multicolumn{3}{|c|}{$I S F=1,45$} & \multicolumn{3}{|c|}{$I S F=1,7$} & \multicolumn{3}{|c|}{$I S F=1,9$} \\
\hline & $\begin{array}{l}\text { Popu- } \\
\text { lation }\end{array}$ & $\begin{array}{c}\text { Nombre } \\
\text { de } \\
\text { migrants } \\
\text { nets }\end{array}$ & $\begin{array}{c}\text { Migration } \\
\text { nette } \\
\text { cumulée }\end{array}$ & $\begin{array}{l}\text { Popu- } \\
\text { lation }\end{array}$ & $\begin{array}{c}\text { Nombre } \\
\text { de } \\
\text { m1grants } \\
\text { nets }\end{array}$ & $\begin{array}{l}\text { Migration } \\
\text { nette } \\
\text { cumulée }\end{array}$ & $\begin{array}{l}\text { Popu- } \\
\text { lation }\end{array}$ & $\begin{array}{c}\text { Nombre } \\
\text { de } \\
\text { migrants } \\
\text { nets }\end{array}$ & $\begin{array}{c}\text { Migration } \\
\text { nette } \\
\text { cumulée }\end{array}$ & $\begin{array}{l}\text { Popu- } \\
\text { lation }\end{array}$ & $\begin{array}{c}\text { Nombre } \\
\text { de } \\
\text { migrants } \\
\text { nets }\end{array}$ & $\begin{array}{c}\text { Migration } \\
\text { nette } \\
\text { cumulée }\end{array}$ & $\begin{array}{l}\text { Popu- } \\
\text { lation }\end{array}$ & $\begin{array}{c}\text { Nombre } \\
\text { de } \\
\text { migrants } \\
\text { nets }\end{array}$ & $\begin{array}{l}\text { Migration } \\
\text { nette } \\
\text { cumulée }\end{array}$ \\
\hline 1986 & 6623 & 0 & 0 & 6623 & 0 & 0 & 6623 & 0 & 0 & 6624 & 0 & 0 & 6624 & 0 & 0 \\
\hline 1991 & 6782 & 0 & 0 & 6801 & 0 & 0 & 6815 & 0 & 0 & 6838 & 0 & 0 & 6856 & 0 & 0 \\
\hline 1996 & 6837 & 1 & 1 & 6895 & 0 & 0 & 6939 & 0 & 0 & 7012 & 0 & 0 & 7071 & 0 & 0 \\
\hline 2001 & 6837 & 12 & 39 & 6914 & 3 & 4 & 6992 & 0 & 0 & 7123 & 0 & 0 & 7228 & 0 & 0 \\
\hline 2006 & 6837 & 19 & 118 & 6914 & 10 & 40 & 6998 & 4 & 7 & 7182 & 0 & 0 & 7331 & 0 & 0 \\
\hline 2011 & 6837 & 27 & 237 & 6914 & 19 & 115 & 6998 & 12 & 49 & 7204 & 0 & 0 & 7399 & 0 & 0 \\
\hline 2016 & 6837 & 39 & 408 & 6914 & 29 & 239 & 6998 & 22 & 137 & 7204 & 8 & 26 & 7436 & 0 & 0 \\
\hline 2021 & 6837 & 52 & 642 & 6914 & 41 & 421 & 6998 & 33 & 278 & 7204 & 17 & 93 & 7436 & 4 & 6 \\
\hline 2026 & 6837 & 64 & 938 & 6914 & 52 & 661 & 6998 & 43 & 473 & 7204 & 27 & 209 & 7436 & 13 & 52 \\
\hline
\end{tabular}

Notes : 1. Le niveau de migration nette récent, qui s'approche du niveau d'équilibre (migration nette = 0), est maintenu jusqu'à 1 'apparition du déclin.

2. Le sigle ISF désigne l'indice synthétique de fécondité. 
Graphique 3

Migration nette nécessaire selon divers niveaux de fécondité. Québec, 1996-2026

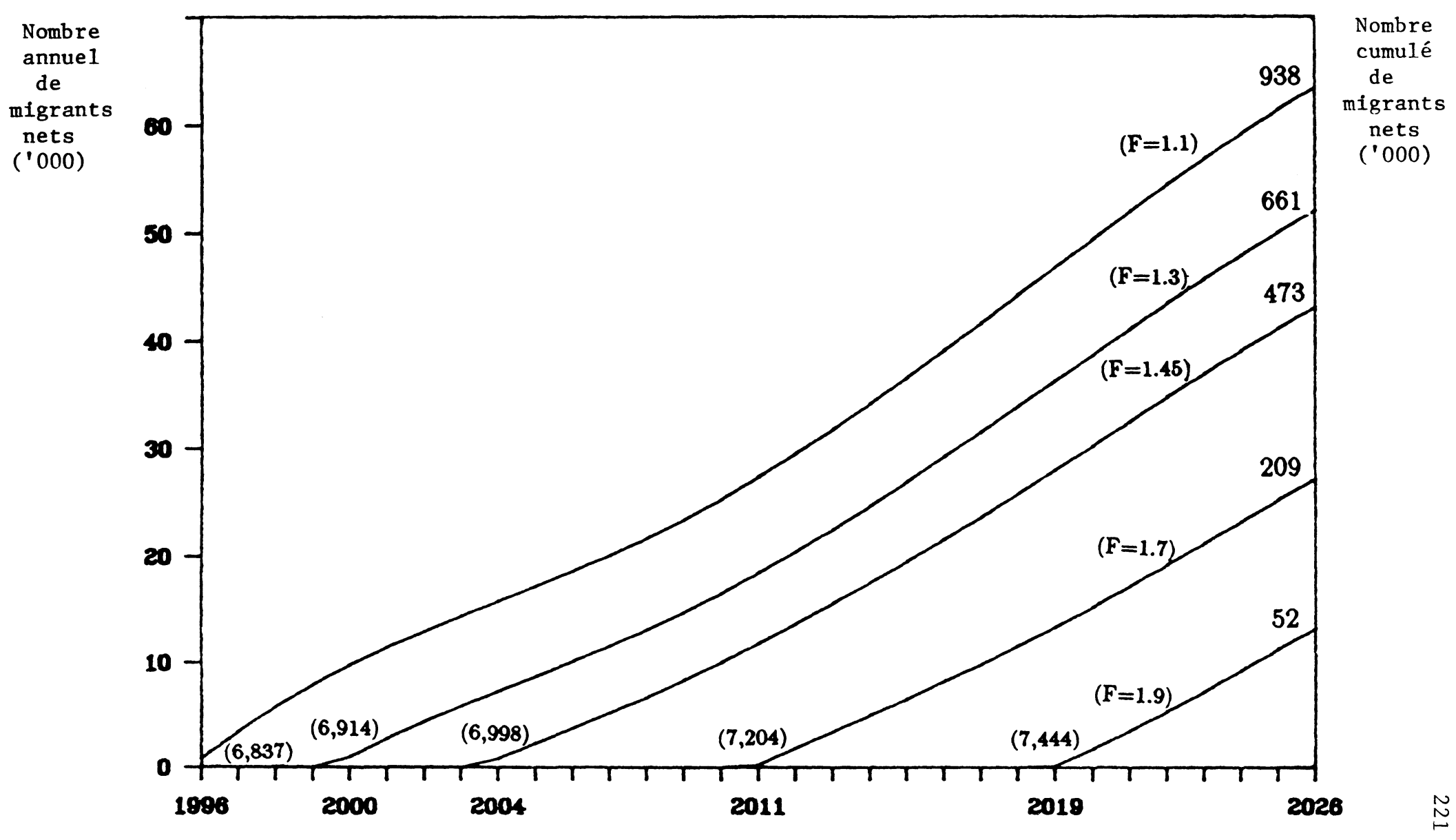

Source: Simulations effectuées par la Section des projections, Division de la démographie, Statistique Canada. 
Le Québec semble donc devancer le Canada et les autres provinces sur la voie de la décroissance démographique.

\subsection{Evolution de la population avec migration nette nulle}

Une autre façon de présenter les résultats consiste à illustrer, selon les divers niveaux de fécondité retenus, l'évolution de la population québécoise en l'absence d'accroissement migratoire (ce qui, répétons-le, correspond grosso modo à la situation actuelle). C'est ce que fait le graphique 4. L'année où le taux d'accroissement de la population devient négatif $y$ est indiquée entre parenthèses.

La décroissance apparaît inévitable - puisque la fécondité est toujours sous le seuil de remplacement des générations - avant 1 a fin du siècle dans 1 'hypothèse la plus faible, vers 2026 dans $1 a$ plus favorable. De façon générale, chaque gain de 0,2 enfant par femme retarde la décroissance d'environ cinq années additionnelles.

\section{CONCLUSION}

Quel que soit le scénario de fécondité qui prévaudra dans l'avenir, s'il se situe à l'intérieur de l'éventail présenté ici, il entrânera à plus ou moins brève échéance une décroissance de la population québécoise. Selon ces scénarios, la taille maximale de la population du Québec n'atteindrait jamais le cap des huit millions d'habitants. Et même, dans 1 'hypothèse de fécondité la plus faible (indice de 1,1 ), la population serait revenue en 2026 au niveau qui était le sien en 1971.

Nous avons vu que, dans l'hypothèse d'une fécondité en dessous du seuil de remplacement des générations, la migration est appelée à jouer un rôle primordial dans 1 'avenir de la société québécoise. Si la décroissance éventuelle devait être perçue comme étant une situation non souhaitable, donc à éviter, la société serait confrontée à des choix difficiles. Entre favoriser la fécondité d'une part et accueillir un nombre sans cesse croissant d'immigrants d'autre part, dépassant peut-être les capacités d'accueil actuelles, il existe toute une gamme d'options mitoyennes combinant les deux facteurs de croissance, et qu'il y aurait lieu d'explorer. 
Graphique 4

Évolution projetée de la population du Québec avec migration nette nulle, selon cinq hypothèses de fécondité

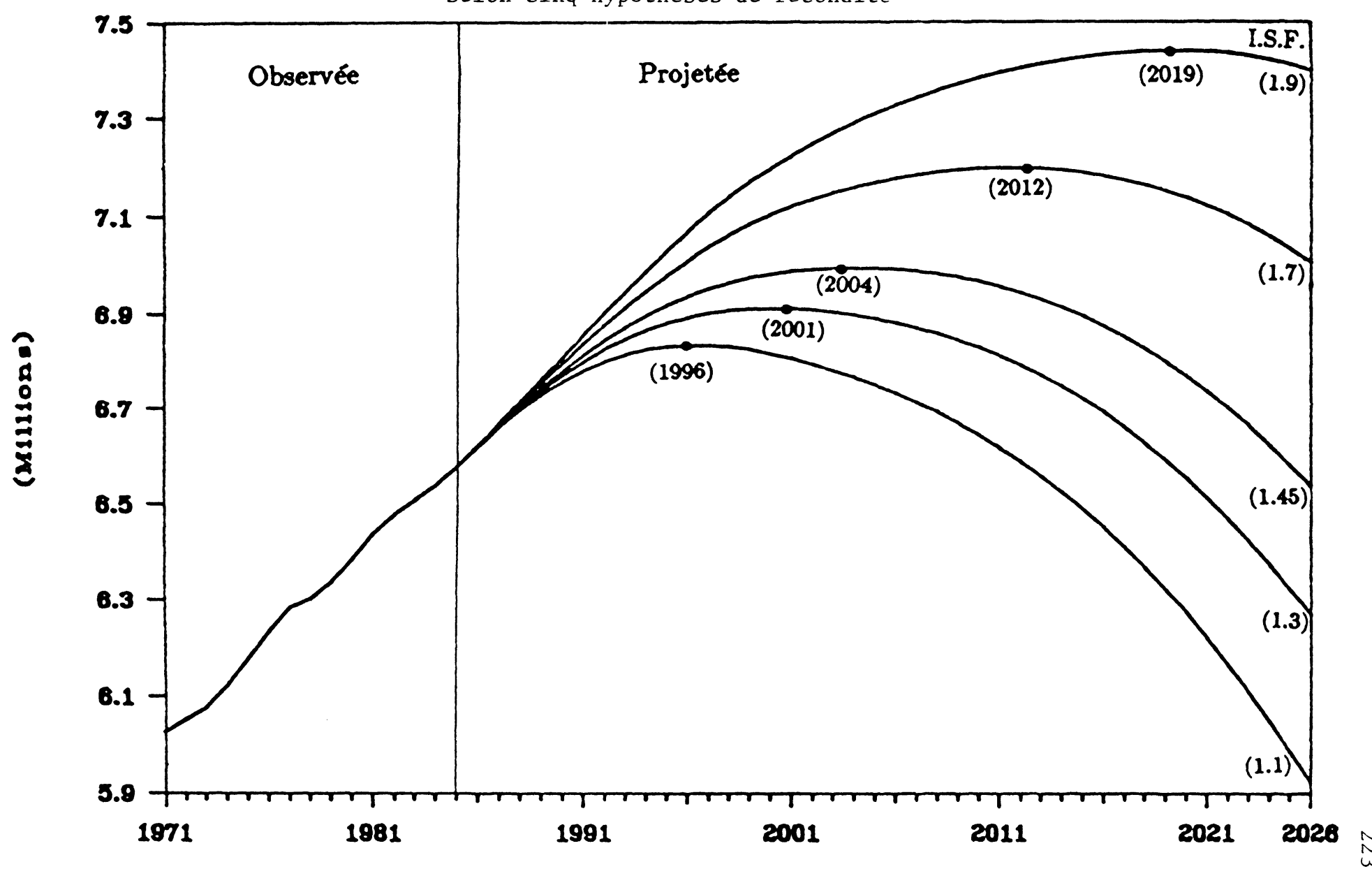

Source : Estimations annuelles postcensitaires de la population. Canada, provinces et territoires au ler juin 1985, volume 3, catalogue 91-210, Statistique Canada, pour 1971-1984; pour les hypothèses d'avenir, voir le texte. 
Nous $n$ 'avons pas fait la distinction entre immigration et émigration, ni entre migration interprovinciale et internationale. Seule la migration totale nette a été prise en compte ici. Si les échanges migratoires avec les autres provinces devaient plus ou moins s'équilibrer, le nombre d'immigrants internationaux devrait être supérieur aux chiffres de migration nette pour compenser 1 'émigration des Québécois vers I'étranger.

En 1981, huit pour cent de la population du Québec (un peu plus de $500 \mathrm{mille} \mathrm{Québécois)} \mathrm{étaient} \mathrm{nés} \mathrm{à} \mathrm{l'étranger.} \mathrm{En} \mathrm{2026,}$ cette proportion pourrait être beaucoup plus forte. Même alors, à cet égard, le Québec sera sans doute encore loin derrière l'Ontario, dont la population née à l'étranger comptait déjà en 1981 pour près du quart de la population totale. 


$$
\text { RESUME - SUMMARY - RESUMEN }
$$

\section{PERREAULT Jeanine, COSTA Rosalinda et GEORGE M.V. - LA DECROISSANCE DE LA POPULATION DU QUÉBEC : SI ON PEUT LA PRÉVOIR, PEUT-ON LA PREVENIR ?}

La fécondité du Québec est la plus faible parmi toutes les provinces canadiennes, et selon toute vraisemblance la population du Québec s'achemine vers la décroissance. Dans cet article, les auteurs tentent d'expliciter certains des scénarios possibles qui pourraient empêcher cette décroissance. Dans un premier temps, ils présentent brièvement les tendances actuelles et précisent les hypothèses retenues pour la simulation. Puis, ils examinent les résultats en situant la décroissance dans le temps et en définissant $I^{\prime}$ ordre de grandeur de la population maximale. Et finalement, ils présentent certains des scénarios de rechange qui pourraient empêcher la décroissance.

PERREAULT Jeanine, COSTA Rosalinda and GEORGE M.V. - QUEBEC'S POPULATION DECLINE: IF IT IS FORESEEABLE, IS IT AVOIDABLE ?

Quebec's fertility is the lowest in Canada, and it is most likely that its population is heading for a decline. In this paper, the authors try to make explicit some of the scenarios which could prevent this decline. They first briefly present the current trends and specify the assumptions used in the simulation. Then, they analyse the results by investigating the onset of the decline and the maximum size of the population. And finally, they present some of the replacement scenarios which may prevent the decline.

PERREAULT Jeanine, COSTA Rosalinda y GEORGE M.V. - EL DECRECIMIENTO DE LA POBLACIÓN DEL QUEBEC : PODEMOS PREVERLA, PODEMOS PREVENIRLA ?

La fecundidad del Québec es la más baja entre todas las provincias canadienses y evidentemente va hacía un decrecimiento. En este artículo, los autores tratan de explicar algunos escenarios posibles para imperdirlo. Presentan brevemente en primer lugar las tendencias actuales e indican las hipótesis retenidos para la simulación. Examinan luego los resultados ubicando el decrecimiento en el tiempo y definiendo la magnitud de la despoblación máxima. Finalmente, presentan algunos escenarios de recambio que podrían impedir dicho decrecimiento. 